\section{Alternative pharmacological treatment options for agitation in Alzheimer's disease}

\author{
Francesco Panza, ${ }^{1,2}$ Vincenzo Solfrizzi, ${ }^{3}$ \\ Bruno P. Imbimbo, ${ }^{4}$ Andrea Santamato, ${ }^{5}$ \\ Madia Lozupone, ${ }^{1}$ Giancarlo Logroscino, $0^{1,2}$ \\ ${ }^{1}$ Neurodegenerative Disease Unit, \\ Department of Basic Medicine, \\ Neuroscience, and Sense Organs, \\ University of Bari Aldo Moro, Bari; \\ 2Department of Clinical Research in \\ Neurology, University of Bari Aldo Moro, \\ Pia Fondazione Cardinale G. Panico, \\ Tricase (LE); ${ }^{3}$ Geriatric Medicine-Memory \\ Unit and Rare Disease Centre, University \\ of Bari Aldo Moro, Bari; ${ }^{4}$ Research \& \\ Development Department, Chiesi \\ Farmaceutici, Parma; ${ }^{5}$ Physical Medicine \\ and Rehabilitation Section, University \\ Hospital of Foggia, Foggia, Italy
}

\section{Abstract}

In patients with dementia and Alzheimer's disease (AD), treatment of neuropsychiatric symptoms (NPS) is a major concern in the management of these devastating diseases. Among NPS in AD, agitation and aggression are common with earlier institutionalization, increased morbidity and mortality, and greater caregiver burden. Pharmacological treatments for AD-related agitation, specifically off-label use of atypical antipsychotics, showed only modest improvements, with increased side-effect burden and risk of mortality. Non-pharmacological treatment approaches have become the preferred firstline option. However, when such treatments fail, pharmacological options are often used. Therefore, there is an urgent need to identify effective and safe pharmacological treatments for agitation/aggression in $\mathrm{AD}$ and dementia. Unfortunately, progresses have been slow, with a small number of methodologically heterogeneous randomized controlled trials (RCTs), with disappointing results. However, evidence coming from recently completed RCTs on novel or repositioned drugs (mibampator, dextromethorphan/quinidine, cannabinoids, and citalopram) showed some promise in treating agitation in $\mathrm{AD}$, but still with safety concerns. Further evidence will come from ongoing Phase II and III trials on promising novel drugs for treating these distressing symptoms in patients with $\mathrm{AD}$ and dementia.

\section{Introduction}

Neuropsychiatric symptoms (NPS) in dementia, ${ }^{1,2}$ previously denominated as behavioral and psychological symptoms of dementia, are often more distressing, impairing, and costly than cognitive symptoms, representing a major health burden for older adults. ${ }^{3}$ NPS varied according to dementia subtype and severity and induced marked disability in functional status, increasing, prevalently, the distress of the caregivers of patients with dementia. NPS in Alzheimer's disease (AD) are thought to reflect one or more types of central nervous system (CNS) dysfunction. Among these CNS alterations, central synaptic or circuit disconnections in frontal-subcortical and corticocortical networks, dysfunction in ascending monoaminergic systems involving serotonin, norepinephrine, or dopamine neurons, and glutamate-mediated excitatory neurotoxicity may have a role on NPS in AD.,4,5 These CNS dysfunctions may occur concurrently and mediate synergistically NPS onset.

A better knowledge of neuropsychiatric pattern of the different forms of dementia could affect significantly treatment approaches. ${ }^{6}$ Furthermore, NPS can manifest early in the course of neurodegenerative diseases and mild behavioral impairment (MBI) has been proposed as a diagnostic construct aimed to identify patients with an increased risk of developing dementia, but who may or may not have cognitive symptoms. ${ }^{7}$ Very recently, the NPS Professional Interest Area of the International Society to Advance Alzheimer Research and Treatment (ISTAART) proposed research diagnostic criteria for $\mathrm{MBI}$, as an extension of the preexisting MBI construct to include, but not mandate cognitive impairment, including mild cognitive impairment (MCI) in the MBI framework. ${ }^{8}$

Among NPS, agitation is a frequent manifestation of $\mathrm{AD}$, vascular dementia (VaD), frontotemporal dementia (FTD), dementia with Lewy bodies (DLB), and other dementia syndromes, ${ }^{9-11}$ appearing to be a clinically important behavioral complication of dementia that warrants further study. The prevalence of agitation in dementia ranges from 20 to $60 \%$, depending on diagnostic definitions used and the population studied. ${ }^{12,13}$ Agitation differs from psychosis and depression of AD in that it may be conceptualized as a single symptom or a symptom complex. ${ }^{14}$ Agitation refers to emotional distress, excessive psychomotor activity, disruptive irritability, and disinhibition, and while it may include aggressive behaviors, agitation can occur without aggression (i.e., repetitious mannerisms, rocking, or pacing). In fact, some subtypes of agitation that may have important clinical differences were also characterized, including physical versus verbal and aggressive
Correspondence: Francesco Panza, Neurodegenerative Disease Unit, Department of Basic Medicine, Neuroscience, and Sense Organs, University of Bari Aldo Moro, Bari, Italy and Department of Clinical Research in Neurology, University of Bari Aldo Moro, Pia Fondazione Cardinale G. Panico, Tricase (LE), Italy.

E-mail: geriat.dot@geriatria.uniba.it

Contributions: FP and VS contributed equally to this work

Key words: Agitation; aggression; Alzheimer's disease; dementia; antipsychotics; antidepressants.

Received for publication: 28 July 2015.

Revision received: 8 October 2015.

Accepted for publication: 12 October 2015.

This work is licensed under a Creative Commons Attribution-NonCommercial 4.0 International License (CC BY-NC 4.0).

(C) Copyright F. Panza et al., 2015

Licensee PAGEPress, Italy

Geriatric Care 2015; 1:5460

doi:10.4081/gc.2015.5460

versus non-aggressive types. ${ }^{15}$ Agitation in dementia often co-occurs with psychosis and depression. There is substantial evidence that verbal agitation is associated with depression, and there may be some relationship to delusions. ${ }^{14,16}$ Psychosis, particularly delusions, and depression occur with increased frequency in aggressive patients and may be a causative factor of agitation/aggression. ${ }^{17}$ Agitation and aggression have frequently been termed dysexecutive symptoms because of their relationship to executive or higher-order loss of behavioral control. ${ }^{18,19}$

At present, there is no consensus on a commonly accepted definition of agitation, with no widespread agreement on what elements should be included in the syndrome. ${ }^{20}$ Historically, a formalized definition of agitation was proposed as inappropriate verbal, vocal, or motor activity that is not judged by an outside observer to be an obvious outcome of the needs or confusion of the individual. ${ }^{15}$ However, very recently, the International Psychogeriatric Association (IPA) formed an Agitation Definition Working Group (ADWG) with a group of experts to develop a provisional consensus definition of agitation in patients with cognitive disorders broadly defined as: i) occurring in patients with a cognitive impairment or dementia syndrome (e.g., AD, FTD, DLB, VaD, other dementias, or a predementia cognitive impairment syndrome such as $\mathrm{MCI}$ or other cognitive disorders); ii) exhibiting behavior consistent with emotional distress; iii) manifesting excessive motor activity, ver- 
bal aggression, or physical aggression; and iv) evidencing behaviors severe enough to cause excess disability and not solely attributable to another disorder (psychiatric, medical, or substance-related) or a suboptimal care condition. ${ }^{20}$ This provisional definition has been developed to advance interventional and non-interventional research of agitation in patients with cognitive impairment.

Finally, given that agitation in dementia may be conceptualized as a single symptom or a symptom complex, ${ }^{14}$ emerging diagnostic constructs for neuropsychiatric syndromes in dementia are drawn from and have theoretical congruence with nondementia diagnoses in psychiatry (e.g., psychotic disorders, or affective disorders). In a large sample of communitydwelling individuals and those residing in nursing homes with late-onset $\mathrm{AD}$, a principal components analysis of the neuropsychiatric inventory (NPI) ${ }^{21}$ identified four interpretable neuropsychiatric endophenotypes: behavioral dyscontrol (euphoria, disinhibition, aberrant motor behavior, and sleep and appetite disturbances), psychosis (delusions and hallucinations), mood (depression, anxiety, and apathy), and agitation (aggression and irritability), with scores on the four components associated with severity of cognitive impairment. ${ }^{22}$ Higher behavioral dysfunction, agitation, and mood component scores were associated with lower age at onset. ${ }^{22}$ More recently, the Behavioural Subgroup of the European Alzheimer's Disease Consortium (EADC) has performed a factor analysis of the NPI in a homogeneous sample of patients with $\mathrm{AD}$, analyzing the largest $\mathrm{AD}$ population ever studied for this purpose. ${ }^{23}$ The Behavioural Subgroup of the EADC identified four separate neuropsychiatric syndromes: affective, apathetic, psychotic, and hyperactive, ${ }^{23}$ providing also evidence of the relative consistency of neuropsychiatric syndromes across dementia subtypes, age and gender. ${ }^{24}$ In particular, the hyperactive syndrome included AD patients with agitation, euphoria, disinhibition, irritability, and aberrant motor behavior, ${ }^{23}$ stressing the importance of thinking about neuropsychiatric syndromes instead of separate NPS in AD patients.

\section{Current pharmacological approaches to the treatment of agitation in Alzheimer's disease}

With both the approaches, single symptom or neuropsychiatric syndrome, agitation is common, persistent, and very difficult to treat. ${ }^{25-28}$ In recent years, a number of pharmacological and psychosocial approaches have proven inadequate, and antipsychotics, antide- pressants, acetylcholinesterase inhibitors, anticonvulsants, and other classes of drugs have been used for treating agitation in $\mathrm{AD} .^{27,28}$ The US Food and Drug Administration (FDA) has not yet approved any medication for treating agitation associated with dementia and $\mathrm{AD}$, while in the European Union, only risperidone, an atypical antipsychotic, is indicated for the short-term management of persisting and severe aggression in $\mathrm{AD}$ patients. In Australia, the regulatory authority, the Pharmaceutical Benefits Advisory Committee (PBAC), indicates risperidone for the treatment of psychotic symptoms and aggression with unsuccessful non-pharmacological methods.

As a result, most agents are used off-label. ${ }^{29}$ In particular, both conventional and atypical antipsychotics are used to treat NPS and agitation associated with $\mathrm{AD}$ and dementia, and offlabel use of atypical antipsychotics (aripiprazole, olanzapine, quetiapine, and risperidone) has significantly increased over the past two decades. Atypical antipsychotics, mainly risperidone, have the best evidence for shortterm efficacy (6-12 weeks), although metaanalyses have not indicated significant benefit for non-aggressive symptoms of agitation..$^{30,31}$ However, in a meta-analysis of 10-12 week randomized controlled trials (RCTs) $(\mathrm{N}=5110)$ of all-cause dementia, death occurred in $3.5 \%$ of dementia subjects treated with atypical antipsychotics and $2.3 \%$ of controls, resulting in a number needed to harm of 100 (range 531000 ) and a number needed to treat of $4-12 .^{32}$ In 11 RCTs of olanzapine and risperidone, $2.2 \%$ drug-treated patients experienced cerebrovascular adverse events compared with $0.8 \%$ placebo-treated patients..$^{33}$ Other observational studies reported increased mortality in older demented patients exposed to either conventional or atypical antipsychotics, ${ }^{34,35}$ with some notable exceptions. ${ }^{36,37}$ Consequently, in April 2005, the US FDA issued a black-box warning for atypical antipsychotics in the treatment of NPS in older patients with dementia because of a 1.6- to 1.7-fold higher death rate in those taking such drugs compared with those taking placebo. In a pivotal RCT of demented patients already on conventional or atypical antipsychotics, three-year survival doubled in those randomized to cease treatment. ${ }^{38}$ On the other hand, in $\mathrm{AD}$ patients with psychosis or agitation that had responded to risperidone therapy for 4 to 8 months, discontinuation of risperidone was associated with an increased risk of relapse. ${ }^{39}$ The large clinical antipsychotic trials of intervention effectiveness Alzheimer's disease (CATIE-AD) RCT showed non-significant treatment effects of three antipsychotics (olanzapine, quetiapine, and risperidone) when compared with placebo. Moreover, time to discontinuation due to intolerable side effects was higher for those taking the antipsychotics versus placebo. ${ }^{40,41}$
However, findings from a recent observational study challenged these findings by showing that after controlling for important risk factors such as cardiovascular risk and severity of psychosis, antipsychotic use was not associated with premature death or increased institutionalization. ${ }^{42}$ These results suggested that, when adjusting for relevant covariates, the presence of NPS, including psychosis and agitation, was linked to poor outcomes rather than the medications themselves. Taken together, these results as well as the limited efficacy of these medications point to significant gaps in our knowledge of the specific neurobiology of these symptoms in $\mathrm{AD}$. Thus, at present, no clear treatment algorithms for agitation in $\mathrm{AD}$ and dementia exist, with the challenge of managing these disturbing NPS using drugs that may pose life-threatening adverse effects.

\section{Alternative pharmacological approaches to the treatment of agitation in Alzheimer's disease}

Therefore, pharmacological and non-pharmacological treatment of agitation is an unmet need in the care of patients with dementia. ${ }^{27,28,43}$ A recent comprehensive review article focused on the status of recent clinical trials for the alternative pharmacological treatment of agitation in $\mathrm{AD} .{ }^{44}$ Emerging evidence on the neurobiological substrates of agitation in AD has led to investigation of repositioned and novel therapeutics for these NPS in dementia as an alternative to antipsychotics. ${ }^{2,4,5}$ The most promising potential pharmacological alternatives include both repositioned and novel drugs such as citalopram, dextromethorphan/quinidine, cannabinoids, scyllo-inositol, brexpiprazole, dronabinol, and prazosin. ${ }^{44}$ At the present time, none of these agents have sufficient clinical evidence in treating agitation in $\mathrm{AD}$ to be recommend using in routine clinical practice. However, two of these drugs showed phase II and III evidence of efficacy for the treatment of agitation/aggression in $\mathrm{AD}$. In a phase III trial, the citalopram for agitation in Alzheimer's disease study (CitAD), 30 -mg daily dose of the selective serotonin reuptake inhibitor citalopram showed a significant decrease in agitation in 186 patients with AD. ${ }^{45}$ However, QTc prolongation and cognitive worsening were observed in the citalopram group,${ }^{45,46}$ representing safety concerns for clinicians. Furthermore, data from the CitAD database were used to assess potential genetic influences on citalopram treatment efficacy for agitation in $\mathrm{AD}$ and treatment outcomes in CitAD showed modest, although statistically significant, influence of genetic variation at serotonin receptor 2A (HTR2A-T102C) and sero- 
tonin receptor 2C (HTR2C-Cys23Ser) loci. ${ }^{47}$ Dextromethorphan/ quinidine (AVP-923; Avanir Pharmaceuticals, Inc., Aliso Viejo, CA, USA), a combination drug containing dextromethorphan, a N-methyl-D-aspartate receptor antagonist and high affinity sigma-1 receptor agonist, and the class I antiarrhythmic agent quinidine, is the first FDA-approved drug for the treatment of pseudobulbar affect. Very recently, in a phase II trial on $220 \mathrm{AD}$ patients with clinically meaningful agitation (ClinicalTrials.gov Identifier: NCT0158 4440), dextromethorphan/ quinidine significantly improved AD-associated agitation, reduced caregiver burden, and was generally well tolerated. ${ }^{48}$

Among novel drugs with completed RCTs, mibampator (LY451395; Eli Lilly S.p.a., Sesto Fiorentino (FI), Italy) is a biarylpropylsulfonamide amino-3-hydroxy-5-methyl-4-isoxazole propionic acid receptor potentiator previously assessed in a phase II trial for its effects on cognition in 181 patients with mild-to-moderate $\mathrm{AD} .{ }^{49}$ While no evidence of efficacy on cognition was found in $\mathrm{AD}$ trials, a significant improvement on the NPS secondary measure was evident, fueling another Phase II trial to further assess the efficacy and safety of mibampator in patients with $\mathrm{AD}$ and clinically significant agitation/aggression symptoms. ${ }^{50} \mathrm{In}$ this double-blind study, 132 outpatients with probable $\mathrm{AD}$ and agitation/aggression, were randomized to receive for 12 weeks $3 \mathrm{mg}$ of oral mibampator or placebo. No significant benefits were observed for mibampator compared to placebo on agitation/aggression symptoms.
However, a secondary outcome ecological measure of behaviors associated with prefrontal cortical dysfunction was positive affected by the drug..$^{50}$ One possible reason for lack of efficacy could be ascribed to the extensive neurodegenerative damage of the frontolimbic networks at the time of study entry, It is likely that drugs may need to be initiated much sooner in the disease process and the field is indeed moving toward earlier diagnosis of dementia-related NPS at or before MCI stage as in the proposed diagnostic construct of MBI. ${ }^{6}$ Among alternative treatment options for the treatment of AD-related agitation, ${ }^{44}$ the phase II trial (ClinicalTrials.gov Identifier: NCT01735630) on scyllo-inositol (ELND005; Transition Therapeutics Inc., Toronto, ON, Canada; Elan Pharmaceuticals, Dublin, Ireland), an inositol stereoisomer that is thought to neutralize toxic $\beta$-amyloid oligomers and prevent them from aggregating, for the treatment of agitation and aggression in 350 patients with moderate to severe AD has ended on May 2015, but results are presently not available. Furthermore, the phase II trial (ClinicalTrials.gov Identifier: NCT01126099) on the $\alpha 1$-adrenoreceptor antagonist prazosin to compare a 12-week course of $8 \mathrm{mg} /$ day of prazosin to placebo followed by 12 weeks of prazosin offered openlabel to treat disruptive agitation in $\mathrm{AD}$ patients has ended on March 2014 instead of July 2015 and such as early termination leaded to small numbers of participants analyzed (20 instead of the planned 120) and technical problems with measurement leading to unreliable or uninterpretable data. On the other hand, one of the two recently completed phase II studies on delta-9-tetrahydrocannabinol (THC, ECP002A) (Clinical Trials.gov Identifiers: NCT01302340 and NCT01608217) provided evidence that for patients with dementia-related NPS, low-dose THC (4.5 mg daily) did not significantly reduce NPS after three weeks, though it is well-tolerated ${ }^{51}$ This RCT tested a very similar molecule to dronabinol, synthetic THC, indicated for the treatment of chemotherapy-induced nausea and vomiting, or for anorexia with weight loss in patients with acquired immunodeficiency syndrome. This was the largest RCT on THC for treating NPS in dementia and unfortunately was negative. Previous studies with THC (2.5$7 \mathrm{mg}$ daily) all reported positive effects on NPS in dementia. ${ }^{52,53}$ The lack of adverse effects may suggest that the dosage was too low. Further studies with higher dosages of oral THC may be required to properly test its potential in the treatment of dementia-related NPS. Ongoing phase II and III trials with novel and repositioned promising drugs may provide alternatives for treating agitation in $\mathrm{AD}$ (Table 1).,44 One of these drugs is brexpiprazole (OPC34712 or Lu-AF41156; H. Lundbeck A/S, Valby, Denmark; Otsuka Pharmaceutical Development \& Commercialization, Inc., Princeton, NJ, USA), a novel molecular compound chemically and structurally similar to aripiprazole and with broad activity across multiple monoamine systems with reduced partial agonism for D2, 5HT1A receptors, and

Table 1. Principal ongoing phase II and III randomized controlled trials for the treatment of agitation/aggression in Alzheimer's disease.

Compound $\quad$ Mechanism of action Estimated enrollment Characteristics Status
(Company)
Clinicallrials.gov
identifier

Brexpiprazole (OPC-34712)

(H. Lundbeck A/S and Otsuka

Pharmaceutical Development \&

Commercialization, Inc.)

NCT01862640

Dopamine D2 receptor partial agonist

Dopamine D2 receptor partial agonist

420 patients with probable $\mathrm{AD}$ and associated agitation (2013-2017)

1 or $2 \mathrm{mg}$ of brexpiprazole administered orally once daily for 12 weeks

A flexible dose of brexpiprazole titrated
Phase III trial (currently recruiting)
Brexpiprazole (OPC-34712)

(H. Lundbeck A/S and Otsuka

Pharmaceutical Development \&

Commercialization, Inc.)

NCT01862640
Brexpiprazole (OPC-34712)

(H. Lundbeck A/S and Otsuka

Pharmaceutical Development \&

NCT01862640
Commercialization, Inc.) $\alpha-2 c$ adren
antagonist

(Orion Corporation, Orion Pharma

Finland and Janssen Pharmaceuticals)

NCT02471196
230 patients with probable $\mathrm{AD}$ and associated agitation (2013-2017) between 0.5 to $2 \mathrm{mg}$ administered orally once daily for 12 weeks

$\begin{array}{ll}\text { Dopamine D2 receptor } & \begin{array}{l}360 \text { patients with probable } \mathrm{AD} \\ \text { partial agonist }\end{array} \\ & \text { and associated agitation } \\ & \text { 2014-2017) }\end{array}$
(2014-2017) $0.5,1$ or $2 \mathrm{mg}$ of brexpiprazole Phase III trial administered orally once (currently recruiting) daily for 8 weeks

Phase III trial (currently recruiting)
AD, Alzheimer's disease.
300 patients with probable $\mathrm{AD}$ and associated agitation (2015-2017)
Low or high dose of ORM-12741 administered orally twice daily for 12 weeks
Phase II trial (currently recruiting) 
enhanced antagonism for 5-HT2A, and $\alpha 1$ adrenoreceptors. ${ }^{54}$ This drug is currently under review by the US FDA as a monotherapy for schizophrenia ${ }^{55}$ and an adjunct to antidepressant medication for major depressive disorder (ClinicalTrials.gov Identifier: NCT01838681). At present, two phase III trials with brexpiprazole are underway for agitation associated with $\mathrm{AD}$ (Table 1). ${ }^{5,44}$ In July 2013, the first phase III trial (ClinicalTrials.gov Identifier: NCT01862640) started to evaluate the safety, efficacy, and tolerability of three months of treatment with 1 and $2 \mathrm{mg}$ of brexpiprazole or placebo given as a fixed dose once daily for the treatment of agitation in 420 patients with probable $\mathrm{AD}$ living in an institutionalized setting (e.g., dementia unit, nursing home, assisted living facility, or other residential care facility) or in a non-institutionalized setting where the subject is not living alone. The primary endpoint is change from baseline in the Cohen-Mansfield agitation inventory (CMAI), but the trial will also measure changes in aggression, global clinical status, and quality of life. In September 2013, the second phase III trial (ClinicalTrials.gov Identifier: NCT01922 258) started enrolling a total of 230 patients and exploring brexpiprazole using a flexible dose titrated between 0.5 to 2 $\mathrm{mg} /$ day depending on efficacy and tolerability in a given patient. Furthermore, a 2-month, observational, rollover trial (ClinicalTrials.gov Identifier: NCT02192554) started in June 2014 to enroll 360 patients with $\mathrm{AD}$-related agitation previously who previously participated in one of the two phase II trials. Finally, ORM-12741 (Orion Corporation, Orion Pharma, Finland and Janssen Pharmaceuticals, Espoo, Finland) is an orally available $\alpha-2 c$ adrenergic receptor antagonist shown to modulate brain activity during stress. 0RM-12741 was originally synthesized as part of a schizophrenia drug discovery program, but after some early clinical studies in Europe it was abandoned for this indication. After seven phase I trials in more than 200 healthy volunteers in Finland, France, and the Netherlands, in June 2015, a phase II trial (ClinicalTrials.gov Identifier: NCT02471196) was started to evaluate the efficacy of three months of treatment in 300 patients with ADrelated agitation/aggression symptoms measured by the NPI clinician rating scale as primary outcome. The estimated study completion date is February 2017.

\section{Conclusions}

Off-label use of atypical antipsychotics for treating dementia-related NPS and agitation showed only modest improvements or no benefits compared to placebo, with evidence of increased risk for cerebrovascular events and mortality ${ }^{2,5}$ Consensus guidelines from medical organizations and working group statements recommended non-pharmacological approaches as first-line treatment, except in emergency situations where these symptoms may lead to imminent danger to patients or caregivers, and/or requiring hospitalization. ${ }^{56-59}$ Options include only caregiver education, training in problem solving, and targeted interventions to induce specific behaviors. ${ }^{41,56} \mathrm{~A}$ recent comprehensive systematic review on sensory, psychological and behavioral interventions for managing dementia-related agitation suggested that person-centred care, communication skills and modified dementia care mapping (all with supervision), sensory therapy activities, and structured music therapies may reduce agitation in care-home dementia residents, with a need for further work on interventions for agitation in people with dementia living in their own homes. ${ }^{60}$ Unfortunately, management of severe, persistent or recurrent agitation/ aggression in AD and dementia unresponsive to non-pharmacological intervention is still a real challenge for clinicians. ${ }^{5}$ Thus, the development of novel drugs targeting NPS and agitation in $\mathrm{AD}$ is urgently needed. To reach this goal, reliable and valid measurement of behavioral symptoms, cohesive and plausible neurobiological models, and advances in neuroimaging and biomarkers to monitor treatment response are all needed. ${ }^{2}$ Unfortunately, progresses has been slow, with a small number of RCTs, characterized by methodological heterogeneity, and with disappointing results. In the next future, several issues must be addressed, including the need for stronger consensus on the syndromal definition of agitation/aggression in $\mathrm{AD}$ and dementia, earlier timing of dug treatment of dementia-related NPS and agitation, choice of primary efficacy outcome measures, the content and timing of the non-pharmacological intervention in placebo and drug arms, concomitant psychotropic medication, and definition of caregivers and their participation. ${ }^{5}$ Furthermore, considering genetic background may be also important. For example, apolipoprotein E (APOE) is the strongest phenotypic modifier in late-onset $\mathrm{AD}$ and is the only genetic marker able to influence drug response and taken into account in phase III RCTs on AD. ${ }^{61,62}$ Moreover, a significant association was found between the APOE 4 allele and an increase in agitation/aggression, hallucinations, delusions, and late-life depression or anxiety in $\mathrm{AD}^{3}$ suggesting a possible role of genetic factors also in RCTs designed for the treatment of NPS in dementia and AD. Evidence coming from recently completed RCTs on novel or repositioned drugs showed promise in treating agitation in $\mathrm{AD}$, although with some safety concerns. ${ }^{44}$ Further evidence will come from ongoing Phase II and III trials on promising novel drugs for treating these distressing symptoms in patients with dementia.

\section{References}

1. Lyketsos CG, Carrillo MC, Ryan JM, et al. Neuropsychiatric symptoms in Alzheimer's disease. Alzheimers Dement 2011;7:532-9.

2. Geda YE, Schneider LS, Gitlin LN, et al. Neuropsychiatric symptoms in Alzheimer's disease: past progress and anticipation of the future. Alzheimers Dement 2013;9:602-8.

3. Panza F, Frisardi V, Seripa D, et al. Apolipoprotein E genotypes and neuropsychiatric symptoms and syndromes in lateonset Alzheimer's disease. Ageing Res Rev 2012;11:87-103.

4. Meeks TW, Ropacki SA, Jeste DV. The neurobiology of neuropsychiatric syndromes in dementia. Curr Opin Psychiatry 2006; 19:581-6.

5. Soto M, Andrieu S, Nourhashemi F, et al. Medication development for agitation and aggression in Alzheimer disease: review and discussion of recent randomized clinical trial design. Int Psychogeriatr 2015;27: 181-97.

6. Kim JM, Lyons D, Shin IS, Yoon JS. Differences in behavioral and psychological symptoms between Alzheimer's disease and vascular dementia: are the different pharmacologic treatment strategies justifiable? Hum Psychopharmacol 2003; 18:215-20.

7. Taragano FE, Allegri RF, Krupitzki H, et al. Mild behavioral impairment. J Clin Psychiatry 2009;70:584-92.

8. Ismail Z, Smith EE, Geda Y, et al. Neuropsychiatric symptoms as early manifestations of emergent dementia: provisional diagnostic criteria for mild behavioral impairment. Alzheimers Dement 2015 [Epub ahead of print].

9. D’Onofrio G, Sancarlo D, Panza F, et al. Neuropsychiatric symptoms and functional status in Alzheimer's disease and vascular dementia patients. Curr Alzheimer Res 2012;9:759-71.

10. Manoochehri M, Huey ED. Diagnosis and management of behavioral issues in frontotemporal dementia. Curr Neurol Neurosci Rep 2012;12:528-36.

11. Boot BP, McDade EM, McGinnis SM, Boeve BF. Treatment of dementia with Lewy bodies. Curr Treat Options Neurol 2013;15:738-64.

12. Lyketsos CG, Lopez 0, Jones B, et al. Mental and behavioral disturbances in dementia: findings from the cache county study on memory in aging. Am J Psych 2000;157:708-14.

13. Margallo-Lana M, Swann A, O'Brien J, et al. Prevalence and pharmacological management of behavioural and psychological symptoms amongst dementia sufferers living in care environments. Int $\mathrm{J}$ Geriatr Psychiatry 2001;16:39-44. 
14. Jeste DV, Meeks TW, Kim DS, Zubenko GS. Research agenda for DSM-V: diagnostic categories and criteria for neuropsychiatric syndromes in dementia. J Geriatr Psychiatry Neurol 2006;19:160:71.

15. Cohen-Mansfield J. Nonpharmacologic interventions for inappropriate behaviors in dementia: a review and critique. Am J Geriatr Psychiatry 2001;9:361-81.

16. Cohen-Mansfield L, Werner P. Longitudinal predictors of nonaggressive behaviors in the elderly. Int J Geriatr Psychiatry 1999; 14:831-44.

17. Deutsch LH, Bylsma FW, Rovner BW, et al. Psychosis and physical aggression in probable Alzheimer's disease. Am J Psychiatry 1991;148:1159-63.

18. Lyketsos CG, Rosenblatt A, Rabins P. Forgotten frontal lobe syndrome or 'executive dysfunction syndrome'. Psychosomatics 2004;45:247-55.

19. Tsoi T, Baillon S, Lindesay J. Early frontal executive impairment as a predictor of subsequent behavior disturbance in dementia. Am J Geriatr Psychiatry 2008; 16:102-8.

20. Cummings J, Mintzer J, Brodaty $\mathrm{H}$, et al. Agitation in cognitive disorders: International Psychogeriatric Association provisional consensus clinical and research definition. Int Psychogeriatr 2015;27:7-17.

21. Hollingworth P, Hamshere ML, Moskvina V, et al. Four components describe behavioral symptoms in 1,120 individuals with lateonset Alzheimer's disease. J Am Geriatr Soc 2006;54:1348-54.

22. Cummings JL, Mega M, Gray K, et al. The Neuropsychiatric Inventory: comprehensive assessment of psychopathology in dementia. Neurology 1994;44:2308-14.

23. Aalten P, Verhey FR, Boziki M, et al. Neuropsychiatric syndromes in dementia. Results from the European Alzheimer Disease Consortium: part I. Dement Geriatr Cogn Disord 2007;24:457-63.

24. Aalten P, Verhey FR, Boziki M, et al. Consistency of neuropsychiatric syndromes across dementias: results from the European Alzheimer Disease Consortium: part II. Dement Geriatr Cogn Disord 2008; 25:1-8.

25. Ballard C, Corbett A. Agitation and aggression in people with Alzheimer's disease. Curr Opin Psychiatry 2013;26:252-9.

26. Small GW. Treating dementia and agitation. JAMA 2014;311:677-8.

27. Gallagher D, Herrmann N. Agitation and aggression in Alzheimer's disease: an update on pharmacological and psychosocial approaches to care. Neurodegener Dis Manag 2015;5:77-83.

28. Nowrangi MA, Lyketsos CG, Rosenberg PB. Principles and management of neuropsychiatric symptoms in Alzheimer's dementia. Alzheimers Res Ther 2015;7:12.
29. Maher AR, Maglione M, Bagley S, et al. Efficacy and comparative effectiveness of atypical antipsychotic medications for offlabel uses in adults: a systematic review and meta-analysis. JAMA 2011;306:1359-69.

30. Ballard C, Howard R. Neuroleptic drugs in dementia: benefits and harm. Nature Rev Neurosci 2006;7:492-500.

31. Schneider LS, Dagerman K, Insel P. Efficacy and adverse effects of atypical antipsychotics for dementia: meta-analysis of randomized, placebo-controlled trials. Am J Geriatr Psychiatry 2006;14: 191-210.

32. Schneider LS, Dagerman KS, Insel P. Risk of death with atypical antipsychotic drug treatment for dementia: meta-analysis of randomized placebo-controlled trials. JAMA 2005;294:1934-43.

33. Herrmann N, Lanctot KL. Do atypical antipsychotics cause stroke? CNS Drugs 2005;19:91-103.

34. Wang PS, Schneeweiss S, Avorn J, et al. Risk of death in elderly users of conventional vs. atypical antipsychotic medications. N Engl J Med 2005;353:2335-41.

35. Kales HC, Valenstein M, Kim HM, et al. Mortality risk in patients with dementia treated with antipsychotics versus other psychiatric medications. Am J Psychiatry 2007;164:1568-76.

36. Barak Y, Baruch Y, Mazeh D, et al. Cardiac and cerebrovascular morbidity and mortality associated with antipsychotic medications in elderly psychiatric inpatients. Am J Geriatr Psychiatry 2007;15:354-6.

37. Raivio MM, Laurila JV, Strandberg TE, et al. Neither atypical nor conventional antipsychotics increase mortality or hospital admissions among elderly patients with dementia: a 2-year prospective study. Am J Geriatr Psychiatry 2007;15:416-24.

38. Ballard C, Hanney ML, Theodoulou M, et al. The dementia antipsychotic withdrawal trial (DART-AD): long-term follow-up of a randomised placebo-controlled trial. Lancet Neurol 2009;8:151-7.

39. Devanand DP, Mintzer J, Schultz SK, et al. Relapse risk after discontinuation of risperidone in Alzheimer's disease. $\mathrm{N}$ Engl J Med 2012;367:1497-507.

40. Schneider LS, Tariot PN, Dagerman KS, et al. Effectiveness of atypical antipsychotic drugs in patients with Alzheimer's disease. N Engl J Med 2006;355:1525-38.

41. Sultzer DL, Davis SM, Tariot PN, et al. Clinical symptom responses to atypical antipsychotic medications in Alzheimer's disease: phase 1 outcomes from the CATIE$\mathrm{AD}$ effectiveness trial. Am J Psychiatry 2008;165:844-54.

42. Lopez OL, Becker JT, Chang YF, et al. The long-term effects of conventional and atypical antipsychotics in patients with proba- ble Alzheimer's disease. Am J Psychiatry 2013;170:1051-8.

43. Gitlin LN, Kales HC, Lyketsos CG. Nonpharmacologic management of behavioral symptoms in dementia. JAMA 2012; 308:2020-9.

44. Antonsdottir IM, Smith J, Keltz M, Porsteinsson AP. Advances in the treatment of agitation in Alzheimer's disease. Exp Opin Pharmacother 2015;16:1649-56.

45. Porsteinsson AP, Drye LT, Pollock BG, et al. Effect of citalopram on agitation in Alzheimer disease: The CitAD randomized clinical trial. JAMA 2014;311:682-91.

46. Drye LT, Spragg D, Devanand DP, et al. Changes in QTc interval in the citalopram for agitation in Alzheimer's disease (CitAD) randomized trial. PLoS One 2014; 9:e98426.

47. Peters ME, Vaidya V, Drye LT, et al. Citalopram for the treatment of agitation in Alzheimer dementia: genetic influences. J Geriatr Psychiatry Neurol 2015 [Epub ahead of print].

48. Cummings JL, Lyketsos CG, Peskind ER, et al. Effect of dextromethorphan-quinidine on agitation in patients with Alzheimer disease dementia: a randomized clinical trial. JAMA 2015;314:1242-54.

49. Chappell AS, Gonzales C, Williams J, et al. AMPA potentiator treatment of cognitive deficits in Alzheimer disease. Neurology 2007;68:1008-12.

50. Trzepacz PT, Cummings J, Konechnik T, et al. Mibampator (LY451395) randomized clinical trial for agitation/aggression in Alzheimer's disease. Int Psychogeriatr 2013;25:707-19.

51. van den Elsen GA, Ahmed AI, Verkes RJ, et al. Tetrahydrocannabinol for neuropsychiatric symptoms in dementia: a randomized controlled trial. Neurology 2015;84:2338-46.

52. Walther S, Schüpbach B, Seifritz E, et al. Randomized, controlled crossover trial of dronabinol, $2.5 \mathrm{mg}$, for agitation in 2 patients with dementia. J Clin Psychopharmacol 2011;31:256-8.

53. Woodward MR, Harper DG, Stolyar A, et al. Dronabinol for the treatment of agitation and aggressive behavior in acutely hospitalized severely demented patients with noncognitive behavioral symptoms. Am J Geriatr Psychiatry 2014;22:415-9.

54. Maeda K, Sugino H, Akazawa H, et al. Brexpiprazole I: in vitro and in vivo characterization of a novel serotonindopamine activity modulator. J Pharmacol Exp Ther 2014;350:589-604.

55. Kane JM, Skuban A, Ouyang J, et al. A multicenter, randomized, double-blind, controlled phase 3 trial of fixed-dose brexpiprazole for the treatment of adults with acute schizophrenia. Schizophr Res 2015;164:127-35. 
56. Lyketsos CG, Colenda CC, Beck C, et al. Position statement of the American Association for Geriatric Psychiatry regarding principles of care for patients with dementia resulting from Alzheimer disease. Am J Geriatr Psychiatry 2006;14: 561-72.

57. Rabins PV, Blacker D, Rovner BV, et al. American Psychiatric Association practice guideline for the treatment of patients with Alzheimer's disease and other dementias. Second edition. Am J Psychiatry 2007;164:5-56.

58. Gauthier S, Cummings J, Ballard C, et al. Management of behavioral problems in
Alzheimer's disease. Int Psychogeriatr 2010;22:346-72.

59. Kales HC, Gitlin LN, Lyketsos CG; Detroit Expert Panel on Assessment and Management of Neuropsychiatric Symptoms of Dementia. Management of neuropsychiatric symptoms of dementia in clinical settings: recommendations from a multidisciplinary expert panel. J Am Geriatr Soc 2014;62:762-9.

60. Livingston G, Kelly L, Lewis-Holmes E, et al. A systematic review of the clinical effectiveness and cost-effectiveness of sensory, psychological and behavioural interventions for managing agitation in older adults with dementia. Health Technol Assess 2014;18:1-226.

61. Gold M, Alderton C, Zvartau-Hind M, et al. Rosiglitazone monotherapy in mild-tomoderate Alzheimer's disease: results from a randomized, double-blind, placebocontrolled phase III study. Dement Geriatr Cogn Disord 2010;30:131-46.

62. Salloway S, Sperling R, Fox NC, et al. Two phase 3 trials of bapineuzumab in mild-tomoderate Alzheimer's disease. N Engl J Med 2014;370:322-33. 